\title{
¿Pasa por YouTube el futuro de la televisión?
}

\section{Is YouTube the future of TV?}

\author{
José María Castillo Pomeda (España) \\ ohcastillo@gmail.com \\ Doctor en Ciencias de la Comunicación Audiovisual \\ Universidad Francisco de Vitoria
}

\section{Resumen}

¿Quién no sabe lo que es YouTube?

Es el cine más grande del mundo, la televisión con más programas, el sueño de cualquier amante de las imágenes, de la narrativa audiovisual... y uno de los mayores negocios del orbe habitado. Ningún otro crece con el vértigo y la insistencia que él, con miles de millones de usuarios de todo el mundo.

El objetivo primordial de este trabajo es tratar de mostrar, Itanto desde un punto de vista tecnológico como desde los campos social y narrativo, sin olvidar el económicol, las características que presenta el "Fenómeno YouTube" en sí mismo y que le presentan como alternativa al medio televisión tradicional. Se avecinan fuertes cambios en el

\section{Summary}

Who doesn't know what YouTube is? Its the world's largest cinema with the most programs, home of the audiovisual narrative... and one of the largest businesses in the world. No other grows with the speed and persistence of YouTube, with its billions of users around the world.

The primary objective of this work is to show, (from a technological point of view as well as from the narrative, social, and economic point of views), the features of the "YouTube phenomenon", which offers an alternative to traditional television media.

Changes on what viewers can see today are taking place, and those changes will happen sooner than expected. 
planteamiento de lo que hoy en día está al alcance de los espectadores. Y van a suceder antes de lo que muchos piensan. El presente es un estudio no experimental, en el que se observan situaciones existentes en nuestro entorno, con una metodología descriptiva de los elementos estudiados y de las interrelaciones que de ellos surjan, encaminada a conseguir unas conclusiones que puedan ser útiles, tanto en el desarrollo de dichos elementos como en la aplicación práctica de los mismos.

Este trabajo se enmarca dentro del proyecto de investigación titulado "La televisión del futuro o el futuro de la Televisión", que el autor está dirigiendo y que es financiado por la Universidad Francisco de Vitoria y el Banco Santander.

Palabras clave: Youtube, comunicación, televisión, narrativas digitales.
This is a non-experimental study, in which we observe existing situations in our environment with a descriptive methodology of the elements studied and the inter-relationships that arise, thus reaching conclusions that might be useful both in the development of such items as in the practical application of them. This work is part of a research project entitled "The television of the future and the future of television", lead by the author and funded by the Universidad Francisco de Vitoria and the Banco Santander.

Key Words: YouTube, communication, television, digital narratives. 


\section{Introducción}

La telefonía móvil y la Red han cambiado casi violentamente nuestras vidas. A la pregunta ¿imaginas hacer la carrera sin ordenador ni Internet?, los alumnos universitarios responden con un gesto de estupor: “sería posible?", parecen pensar, mientras su boca permanece muda.

Un viaje en transporte público nos permite observar que, salvo algún anciano, todos los viajeros se afanan en su smartphone haciendo diversas y parece que urgentes e inaplazables tareas.

Se ha sustituido la reunión o la tertulia por el Whatsapp y las redes sociales. Esto está muy bien porque tenemos muchísimos amigos e incluso seguidores. Hace poco vi cómo un futbolista se enorgullecía de lo mucho que revisaba el texto de sus mensajes en Twitter, porque "tiene 5 millones de seguidores y si pone mal una coma... ¡menuda responsabilidad!”.
Este, al menos, cuida la ortografía de sus autorizados comentarios.

Hoy es posible ganarse la vida colgando en YouTube los vídeos de cualquier cosa (repito: cualquier cosa) que cualquiera haga delante de una cámara.

De entre todas las revoluciones que nos ha traído la digitalización, la aparición de YouTube quizá sea la más trascendente, ya que podemos decir que se ha convertido en nuestra memoria audiovisual.

\section{Los comienzos}

Aunque su gestación es un tanto confusa, parece ser que todo tuvo su origen en el deseo de compartir vídeos de los alegres participes de una fiesta en San Francisco, aunque también corre la especie de que la idea surgió por imitación de un portal de citas llamado HotorNot. 
Sea como fuere, lo cierto es que Chad Hurley, Steve Chen y Jawed Karim, tres ex empleados de PayPal, el intermediario de pagos por Internet, decidieron independizarse y emprender su propio negocio.

El 15 de febrero de 2005 registraron un dominio que, ante el asombro de la propia empresa, tuvo tal éxito que año y medio más tarde recibieron de Google 1.650 millones de dólares por el "invento" que se había hecho con el favor de los internautas, haciendo fracasar a todas las imitaciones que rápidamente surgieron por doquier, incluida la del propio buscador omnipotente.

\section{El éxito y las cifras}

Desde ese momento, bajo el paraguas de Google e integrado en él como uno de sus servicios, ha seguido creciendo sin cesar. Las cifras son mareantes: ya en 2006 se visualizaron 100 millones de vídeos y se subieron a diario más de 65.000. Oficiosamente se maneja la cifra de 200 millones de beneficio en ese primer año para Google.

- El propio YouTube nos brinda sus estadísticas:

- YouTube tiene más de mil millones de usuarios (para que te hagas una idea, equivaldría a un tercio de todos los usuarios de Internet), y cada día se ven cientos de millones de horas de vídeos y se generan miles de millones de reproducciones.

- Si analizamos YouTube en general (aunque valdría con fijarnos solo en la versión para dispositivos móviles), nos daremos cuenta de que muchas más personas con edades comprendidas entre los 18 y los 49 años deciden ver YouTube en lugar de la TV por cable en Estados Unidos.

- El número de horas que la gente dedica a ver vídeos (lo que se conoce como "tiempo de visualización") en YouTube ha aumentado un $60 \%$ de un año a otro. Es el crecimiento más rápido que hemos visto en tan solo 2 años.

- El número de horas que la gente dedica a ver vídeos de YouTube al día ha ascendido un 40\% desde marzo de 2014.

- El número de usuarios que llegan a YouTube por su página principal (una acción parecida a la de encender la TV) se ha triplicado de un año a otro. 
- El 80\% de las reproducciones de YouTube tienen lugar fuera de EE. UU.

- YouTube tiene versiones locales disponibles en más de 70 países.

- Puedes navegar por YouTube en un total de 76 idiomas distintos (en otras palabras: puede entendernos el 95\% de todos los usuarios de Internet). (YouTube 2015)

Tal es la popularidad de la plataforma, que artistas como Justin Bieber, Esmee Denters, Cimorelli, Greyson Chance, Sam Tsui, Clarice Pompegco, Boyce Avenue, Timeflies, Cody Simpson, Austin Mahone, Max Schneider, Tiffany Alvord, Pablo Alborán, Xuso Jones o Tay Zonday, entre otros, han llegado a la fama gracias a demostrar su talento en este espacio. YouTube se ha convertido en una ventana abierta al mundo, y muchas veces, son los propios usuarios los que someten a examen ese contenido. "Lo que antes era simplemente audiencia, ahora decide qué es importante, y no un pequeño grupo de personas" (O'Reilly, 2005) La intuitiva y sencilla difusión de sus vídeos es otra de las claves de su popularidad, puesto que permite insertar cualquiera de ellos tan solo con la URL o el código HTML en una página web, una red social, un blog, etc. Técnicamente, lo único que necesita un usuario para utilizar la plataforma es un navegador, una conexión mínima de 500kbps y el Adobe Flash Player.

Es compatible con los formatos .MOV, .MPEG4, .AVI, .WMV, .FLV., MPEGPS, 3GPP y WebM. Además, se permiten $128 \mathrm{G}$ y hasta 11 horas de vídeo, aunque sin tener una cuenta verificada el limite serán 15 minutos. La calidad del contenido varía en función de la conexión, pudiendo visualizarse en 240, 360, 480 pixeles con una definición estándar o en alta definción, 720 o 1080 pixeles. No obstante, en los últimos años se han implementado mejoras con el objeto de integrarse en el curso del avance tecnológico contemporáneo, de tal manera que permite reproducir videos a $4 \mathrm{~K} \mathrm{u} 8 \mathrm{~K} \mathrm{e}$ incluso contempla 1080p a 60 fotogramas por segundo. Ya se pueden ver vídeos en $4 \mathrm{~K}$ (hasta $60 \mathrm{fps}$ ) y $8 \mathrm{~K}$, además de espectaculares grabaciones en $360^{\circ}$ (de 24, $25030 \mathrm{fps}$ ) que consumen de cuatro a cinco veces más de ancho de banda.

Según revela la propia plataforma, cuenta con más de mil millones 
de usuarios únicos. Al día se consumen cientos de millones de horas, una cifra que aumenta el 50\% cada año. Además, los internautas suben 300 horas por minuto entre los 75 países en los que está disponible, lo que genera alrededor de $6.000 \mathrm{mi}-$ llones de horas de vídeo mensuales.

El diario web marketingdirecto.com, afirma que el $53 \%$ de los jóvenes entre 16 y 24 años adoran YouTube, una plataforma que es utilizada por el 16\% de los internautas varias veces al día y por el $28 \%$ varias veces a la semana. Puntualizando la ubicación de los usuarios, el $80 \%$ de los mismos procede de fuera de Estados Unidos, a pesar de que en este país la plataforma de Google reúne a más jóvenes con edades comprendidas entre los 18 y 38 años que cualquier cadena de televisión (marketingdirecto.com, 2014). En España, se consumió una media de 3,34 horas a la semana en un promedio de 4,4 días en 2014. El perfil del usuario habitual corresponde a la edad comprendida entre $18-30$ años con un $37 \%$, aunque un $34 \%$ de los usuarios está entre los 31-39 (IAB Spain, 2014).

Según María Ferreras, directora de alianzas estratégicas de YouTube, "el grueso de la audiencia de la plataforma se concentra entre los 18 y los 44 años. Los usuarios entre 13 y 17 son muy activos y su consumo es muy alto, pero aun así la principal horquilla es de más edad".

Estas cifras, junto con el más de un millón de anunciantes que utilizan la plataforma, les ha permitido invertir en sus creadores. En 2011 se comenzó el proyecto para partners de YouTube, por medio del cual los creadores de contenido podían obtener ingresos con la inserción de publicidad en sus canales con los diferentes formatos de TrueView que veremos en detalle en el epígrafe 7. Los únicos requisitos, especificados en la web de la plataforma, se basan en subir contenido original que no infrinja los derechos de copyright ni de los anunciantes, y no haber sido expulsado del programa en ningún momento. Por ello, entre 2013 y 2014 los ingresos de los partners, que a día de hoy son más de un millón, han crecido un 50\%. Tal ha sido la implicación de Google, que ha invertido en la creación de seis YouTube Spaces, localizados en Nueva York, Berlín, Tokio, Los Ángeles, Sao Paulo y Londres, a los que han acudido 60.000 personas. En estas instalaciones se puede asistir a talleres, eventos, usar las salas 
de producción, colaborar con otros canales, etc.

Alguien que acaba de abrir un canal puede venir a todos los eventos sociales que hay, en los cuales puedes conocer otros youtubers, puedes hablar con gente de la industria, puedes tener información $\mathrm{u}$ obtener clases. Cuando tienes diez mil suscriptores o más, puedes también acceder a la producción, y empezar a utilizar todas las salas que hay, la edición, tener apoyo para producir cosas que quizá por tu cuenta no pudieras... Y cuando tienes más de cien mil y un millón, estas opciones se van ampliando. (LuzuVlogs, 2015)

\section{Internet, ese nuevo visitante}

Pero antes de comenzar a hablar de YouTube y los youtubers, habrá que centrar el tema revisando el medio en que florece, Internet, para llegar a entender en qué condiciones evoluciona y se encuentra actualmente. Según la RAE, Internet es "una red informática mundial, descentralizada, formada por la conexión directa entre computadoras mediante un protocolo especial de comunicación". Esta definición nos plantea un escenario que, desde sus comienzos en 1969, ha tenido una evolución vertiginosa. De hecho, desde la creación de la World Wide Web en 1991 por Berners-Lee, Internet ha experimentado un desarrollo que ningún otro medio de comunicación de masas había conseguido jamás. Mientras que la radio necesitó 38 años para alcanzar la cifra de los 50 millones de usuarios en Estados Unidos, la televisión tardó 13, los ordenadores personales 16, internet lo ha logrado en tan solo cuatro años.

Las herramientas de comunicación, publicación de contenido propio y redes sociales, que fueron el origen de la revolución en el concepto de comunicación, nacieron entre 2003 y 2005: Friendster, primera red social en alcanzar 1 millón de usuarios. Muchas patentes de esta web son usadas hoy en día (2002); MySpace, Linkedin, Skype y WordPress (2003); Facebook, Vimeo y Flickr (2004); YouTube y Dailymotion (2005); Twitter y Tuenti (2006); (2007); Instagram (2010); Google + (2011) y Vine (2013).

Atendiendo a este progreso, cabe señalar que hoy en día, "la innovación tecnológica se dirige a satisfacer las necesidades de unos 
usuarios cada vez más competentes en el uso de tecnologías de la información" (Roig, 2006). De hecho, esa competencia es tal que Marc Prensky remarcó la brecha entre generaciones con los términos: nativo digital e inmigrante digital. El primero hace referencia a las personas que han nacido ya inmersas en la tecnología digital y los nuevos medios; por el contrario, aquellas generaciones que han tenido que adaptarse al cambio, se les considera inmigrantes digitales. (Prensky, 2001).

Es importante considerar ambos términos para definir al usuario principal de Internet, puesto que son los consumidores más proclives a participar en los nuevos fenómenos de la red.

Una vez concretado qué es internet, cómo ha evolucionado y qué herramientas ofrece, podemos establecer los cambios que, gracias a unos consumidores más preparados y a unas herramientas concretas a su disposición, inevitablemente produjeron en la forma de entender la comunicación un aumento de la oferta, fragmentación de la audiencia, producción multiplataforma, el lenguaje, la inmediatez, el acceso, disponibilidad, movilidad, calidad, interactividad e incluso las formas de consumo (Roig, 2006). Este mismo autor, reflexionando sobre el cine y la televisión, realizó una definición muy acertada de las formas de consumo a las que se hacen referencia:

El cine y la televisión convencionales pueden verse actualmente como puntos de entrada familiares a formas de consumo cada vez más híbridas, donde medias emergentes y tradicionales se hallan interrelacionados y donde se desdibujan las barreras entre recepción y emisión, producción y uso, comunicación de masas y comunicación interpersonal, entre lo profesional y lo amateur, entre lo público y lo privado. (Roig, 2006)

Por lo tanto, se puede concretar que "internet, cultura de la libertad, la interacción y la participación, expresada tecnológicamente, crea una plataforma tecnológica que permite ampliar extraordinariamente el intercambio artístico y cultural" (Castells, 2002, p. 8).

Pero quizá lo más impresionante de este nuevo medio es su alcance. Según los últimos datos recogidos en el informe publicado por la Oficina de desarrollo de telecomunicaciones de la UIT, es utilizado por 
casi 3.000 millones de personas a finales del 2014, es decir, el 40\% de la población mundial. Un análisis sesgado regionalmente presenta un $75 \%$ de presencia en Europa y un 65\% en las Américas. En 2010, en África, un 10\% de los hogares tenían acceso a Internet y en cuatro años se ha duplicado.

\section{Publicidad en internet}

La década de los 90 es pródiga en acontecimientos que van a influir decisivamente en el campo publicitario.

Según un estudio elaborado por The Cocktail Analysis para National Geographic Channel, el 54\% de los internautas destaca la llegada de Internet como el evento sociocultural más importante de la década de los 90 en España, seguido del comienzo del uso del teléfono móvil con un $41 \%$, por encima de otros eventos sociopolíticos como la abolición del apartheid en Sudáfrica (25\%), el Tratado de Maastricht (20\%) o la desintegración de la Unión Soviética (16\%). Cuando se les pregunta por el dispositivo más representativo de la época, el $56 \%$ asegura que el Walkman es el icono de los 90, seguido por el móvil (45\%). El Walkman era el objeto más presente en los hogares españoles (83\%), seguido de la mini cadena musical (76\%) y el ordenador de sobremesa (62\%) (Nationalgeographic.es, 2014).

La aparición de las cadenas de televisión privadas supuso una fragmentación de audiencia y todo un territorio por explotar para la publicidad. Además, culturalmente se comienza a reclamar un cambio, que llega con la aparición de Internet y el progreso en la conectividad. Por último, aparecen los buscadores Internet Explorer (1995) y Google (1998).

A estas alturas, la publicidad en la red era de obligada aparición. Puede decirse que nace con el primer email spam el 3 de mayo de 1978, cuando Gary Thuerk envió información sobre una presentación de un producto en California, aunque el nombre de spam no fue aplicado hasta 1994, refiriéndose a un email comercial que no había sido solicitado. Stanford Wallace fue uno de los pioneros en el spam masivo (Spira, 2003, pp. 4-5).

En 1993 aparece el primer sitio Web que cede sus páginas a diferentes marcas para que puedan ofrecer sus productos. Este sitio, llamado Navigator $(\mathrm{CNN})$, cerró tres años más tarde. 
Hay muchas otras fuentes que consideran que el comienzo de la publicidad online llega con la publicación del primer banner el 27 de octubre de 1994. Estos aparecieron en la versión online de la revista estadounidense HotWired (sobre últimas tecnologías). El primero fue para AT\&T, pero posteriormente llegaron marcas como Zimat o Volvo. El tamaño de los primeros banners era de $468 \times 60$ píxeles, y aunque muchos reaccionaron en contra, el hecho es que era la primera vez que una marca podía conocer el número exacto de personas que vieron su anuncio (Wired.com, 2015). En sus inicios, el CTR de los banners (Click Through o Ratio de cliqueo: hace referencia a la eficacia de una pieza publicitaria calculando el porcentaje entre impresiones y clics), estaba mínimo en el 50\%, aunque llegaba a alcanzar cifras que hoy se ven imposibles.

El banner se introdujo por primera vez en Europa, en el Reino Unido en 1995, y fue el periódico británico Electronic Telegraph que inició, con un anuncio de Barclays Bank, su colocación. Entre ese mismo año y el siguiente se extendió el uso de banners animados. Además, comenzó una práctica habitual hoy en día, como es el patrocinio de páginas web. Ese mismo año, en 1996, se producirían cambios en la manera de entender la publicidad online, otorgándole más credibilidad y seriedad en el sector. Uno de ellos será la aparición de Internet Advertising Bureau (IAB), una organización encargada de regular y estandarizar la industria de la publicidad en internet y que actualmente aún es un referente a nivel mundial. Además, Hewlett Packard lanzó el primer banner interactivo, en el cual el usuario podía jugar al ping-pong contra el ordenador.

En España, el primer banner fue insertado en el buscador OZU. Y por último, se empezó a cobrar por clics y no por visitante (CTR), lo que supuso una gran diferencia con otros medios convencionales.

En 1998, se produjo un gran crecimiento de internet a nivel mundial. En España, el sonido comenzó a tener presencia en los banners, concretamente en una campaña que realizó la versión online de Páginas amarillas, gracias al uso de la tecnología "Flash". En ese mismo año también apareció en España un nuevo tipo de banner dinámico programado en HTML a cargo de Audi.

Un año más tarde, en 1999, surgió un nuevo sistema publicitario 
que se basaba en la creación de webs complementarias a las originales, que contenían información acerca de un producto o servicio en particular. En el año 2000 se produjo un evento revolucionario en el mundo de la publicidad en internet. Google presentó $A d W o r d s$, un sistema mediante el cual, a través del uso de palabras clave, el cliente pagaría únicamente cuando los usuarios hicieran clic en el anuncio. De esta manera, las marcas comenzarían a buscar posicionamiento en la web.

Los formatos publicitarios comenzaron a incorporar con éxito en 2001 más vídeos, interactividad, sonidos y otras variables en sus banners. A este tipo de formatos se les referenciará como Rich Media y en ese mismo año comenzaron a destacar entre el resto de formas clásicas. Entre 2004 y 2008, los banners sufrieron una crisis que repercutiría en la tasa de clics. En el 2010, solo el $1,5 \%$ de personas hacen clic en un banner de cada 1000. Además, en ese mismo año, surgió Google Display Network, una plataforma de Google que permitía a las marcas incluir su banner en una red de publishers.

Tres años más tarde, Internet superó a la televisión en inversión publicitaria en Estados Unidos, cosa que ya había logrado en el 2009 en el Reino Unido. En España ocurrió lo mismo con la prensa en el 2012 (lavanguardia.com, 2014).

Pero, ¿cómo está el panorama publicitario en la red actualmente? Rafael Ordozgoiti ya reclamaba en 2010 una coherencia a las marcas entre los frentes off y online, mientras defendía que ambos campos deben trabajarse de forma conjunta, buscando un único posicionamiento (Ordozgoiti, 2010, p. 17). Internet se ha convertido en un punto de encuentro colectivo con franjas de edades muy variadas, exceso de información y en evolución constante. La actividad publicitaria se está desenvolviendo en la época de la inmediatez, del cambio continuo inherente al incesante avance tecnológico, de la comunicación transmedia en un entorno multiplataforma donde el usuario pretende ser único y especial, del intercambio de información y las ganas de aportar y de la digitalización de cualquier empresa o medio. La época en la que cada vez más, "internet es un lugar en el cual informarse, comunicar y divertirse, pero también, es el lugar en donde se deciden muchas compras" (Zunzarren y Gorospe, 2012). 


\section{YouTube como plataforma}

En la década de los 60, Marshall McLuhan -en su libro Understanding Media- afirmó categóricamente: "El mensaje es el medio". Probablemente no contaba entre sus predicciones la aparición de una aplicación tan simple de utilizar y que creciera de forma más que exponencial en tan poco tiempo, como sucedió con YouTube, pero sí sabía muy bien que en algún momento la evolución tecnológica y posterior inserción social de los medios masivos de comunicación llevaría a modificar el curso $\mathrm{y}$ funcionamiento de las relaciones y actividades humanas (De Cicco, 2008, p. 31).

$\mathrm{Si}$ intentamos extraer conclusiones del fenómeno YouTube y de los youtubers, debe existir una investigación previa de su entorno. En este sentido, se pueden diferenciar dos aspectos importantes sobre YouTube: su contexto comunicacional y la personalidad de la plataforma. Uno de los fundamentos de Internet, y más aún de las redes sociales, es la cultura de la participación, que según Jenkins (2006), "está relacionada con una inteligencia colectiva y una convergencia de los medios". Este mismo autor, aparte de situar a los fans como pioneros de una nueva relación con los medios por su proyección participativa, reflexionó sobre la evolución sufrida por la cultura del espectador hasta mudar a la cultura de la participación, donde los contenidos circulan por cientos de plataformas diferentes, en las cuales "los fans y otros consumidores son invitados a participar activamente en la creación y circulación de nuevo contenido" (Jenkins, 2006, p. 290).

El desarrollo de este nuevo concepto, que ha venido a denominarse "Narrativa Transmedia", ha cambiado la forma en la que la gente percibe y ejercita la comunicación. La revolución de YouTube, señalan Burgess y Green, tiene menos que ver con la tecnología y más con cuestiones culturales y políticas, como, por ejemplo, "quién habla y quién escucha; qué compensaciones o recompensas hay para la creatividad y el trabajo y las incertidumbres sobre varias formas de experiencia y autoridad" (Burgess \& Green, 2013). Es decir, los nuevos métodos y herramientas de comunicación facilitan la creación a cualquiera que tenga unos recursos mínimos para su producción. Ya no es necesario ser director de cine o de un programa de televisión para llegar a un público 
considerable, basta con coger una cámara o un smartphone, grabarte y subirlo a YouTube, y es posible que tengas más repercusión que muchos de esos programas televisivos. Burgess y Green (2013), continúan abordando esta idea y afirman que la plataforma de Google ha conseguido perturbar el modelo de negocio de los medios y ha emergido como una alternativa nueva y poderosa.

YouTube se sitúa como un espacio donde el público o las masas están surgiendo desde la base, por lo que los problemas que los conciernen tienen que ver con la rebeldía, la crisis de la experiencia y el colapso del valor cultural. (Burgess y Green, 2013)
Aunque se muestran críticos con la plataforma, reconocen que es un sitio extraño y a la vez maravilloso generado por los usuarios. Esa transformación cultural fue ligada a la Web 2.0, un término acuñado en 2004 en la preparación de una conferencia por Dale Dougherty, el cofundador de O'Reilly Media junto con Tim O’Reilly. El término hacía alusión a la transformación que estaban sufriendo las tradicionales y antiguas herramientas de Internet y la Web 1.0, que se vieron sustituidas por otras totalmente diferentes que reivindicaban la arquitectura participativa, la multidireccionalidad, la libertad de edición y difusión, etc. (García,2003).

Mapa conceptual de la Web 2.0 elaborado por Markus Angermeie

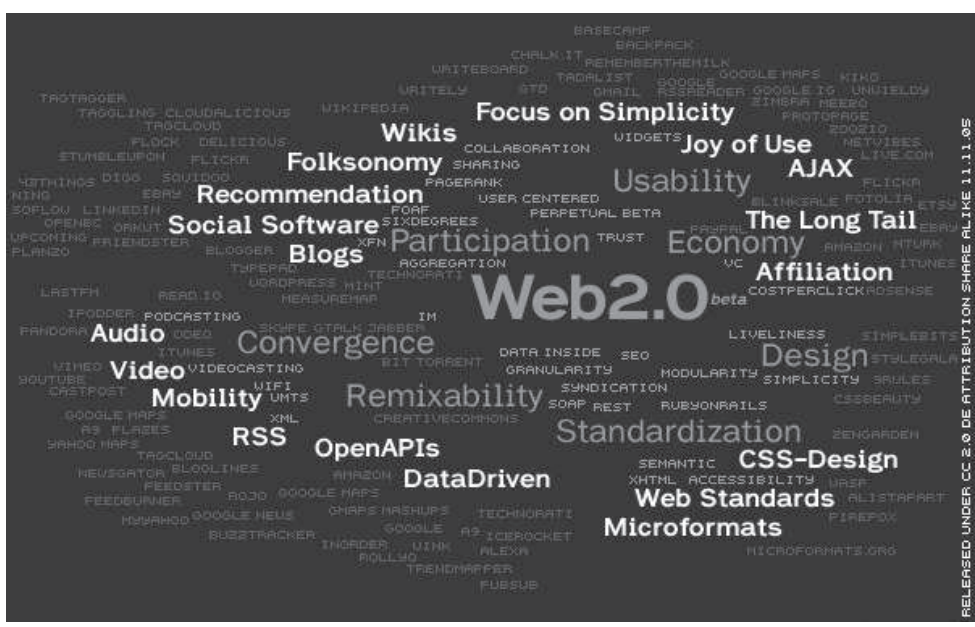

(Fuente: http://museums.wikia.com/wiki/File:Web20map.png) 
Markus Angermeier creó un mapa conceptual sobre esta idea, que fue utilizado después por Dougherty para explicar la Web 2.0. Internet pasó de ser únicamente de entrada, a ser de entrada y salida; de lectura y escritura. El ejemplo más gráfico es comparar Wikipedia frente a Británica Online. Este referente de la Web 1.0 era un diccionario al que los usuarios acudían para consultar ciertos términos; en cambio, con la llegada de Wikipedia, cualquiera podía modificar el contenido de la web e incluir nuevas acepciones y entradas.

Todos tenían la posibilidad de participar en una red de información e inteligencia colectiva. El protagonismo lo recibieron los usuarios, y de esta renovación han surgido conceptos como el marketing viral, los blogs, el coste por clic, las redes sociales, etc. Por tanto, se puede concluir que el nacimiento de YouTube en 2005, y su correspondiente éxito, estuvo respaldado por una cultura participativa y un nuevo concepto de web.

\section{YouTube. Las claves del éxito}

La primera de las claves de su éxito es, sin duda, la sencillez. No es necesario ningún conocimiento técnico para utilizar YouTube, ya que esto limitaría el acceso a usuarios avanzados y reduciría el nivel de audiencia-exposición-participación. Porque no olvidemos que se trata de un canal de comunicación y por lo tanto la audiencia es prioritaria.

El reproductor en línea utiliza Flash o HTML, lo que hace el acceso prácticamente universal a diversos contenidos como vídeos originales, películas completas o secuencias, montajes a base de fotografías, secuencias televisivas, vídeos musicales o tráilers de películas o libros. También los blogs y video blogs aparecen en la plataforma para aumentar su difusión, al igual que las empresas exhiben su publicidad, vídeos corporativos y sus últimos anuncios para que la audiencia aumente y opine.

En esta facilidad para "embeber" o insertar los vídeos en blogs o páginas web se basa la difusión de su contenido, lo que permite que páginas, personales y corporativas puedan añadir con facilidad los vídeos a sus sitios, retroalimentando la difusión y provocando que la audiencia de YouTube haya crecido hasta los niveles que lo ha hecho y 
siga creciendo. Esta inserción puede hacerse de dos formas, usando la aplicación correspondiente en blogs como Wordpress, o a través de código HTML.

El propio usuario puede descubrir contenido interesante investigando en los vídeos relacionados con los que acaba de ver, que aparecen en el margen derecho de la ventana de visionado. Pero además de esto, en su página de perfil, también encuentra recomendaciones que vienen dadas por las reproducciones que se han realizado con anterioridad, creándose así una base de datos de los gustos de cada usuario, en un proceso de retroalimentación. No es necesario comentar las repercusiones del uso de esa base.

El sistema permite la descarga directa al discurso duro del ordenador del usuario y en varias calidades que llegan al $4 \mathrm{~K}$, de momento.

Esto en cuanto a sus funciones como reproductor de vídeos, es decir, como pantalla de visionado, pero no menos importante es su función exhibidora, como elemento o plataforma de comunicación, donde cualquiera puede subir su mensaje y darlo a conocer. YouTube ha eliminado la figura del cineasta que, terminada su obra, era obligado a salvar una carrera de obstáculos y en muchos casos arruinarse, deambulando por las distribuidoras sin conseguir estrenar su película. Ahora la puede exhibir en un cine con millones de espectadores.

\section{Publicidad en YouTube. Estrategias y formatos}

La propia plataforma publica estos datos en su página:

- Los ingresos de los partners han aumentado un 50\% de un año a otro (un nivel de crecimiento que se ha repetido 3 años seguidos).

- El número de canales que ingresan importes de 6 cifras al año gracias a YouTube ha aumentado un $50 \%$ de un año a otro.

- Nos hemos dado cuenta de que nuestros formatos de anuncios de vídeo (como TrueView), han tenido una gran aceptación, y seguiremos contribuyendo a que los profesionales de marketing sigan interactuando con los consumidores de muchas nuevas formas. El número de anunciantes que publican anuncios de vídeo en YouTube ha aumentado más 
del $40 \%$ de un año a otro y, de los 100 más importantes, el gasto medio por anunciante ha crecido más de un $60 \%$ con respecto al año anterior.

- Las 100 marcas principales de todo el mundo han publicado anuncios TrueView el año pasado, y el 95\% de los anunciantes TrueView han ejecutado campañas en diversos dispositivos. También hemos percibido un aumento del número de anunciantes que han adoptado el formato TrueView, ya que el número total aumentó un 45\% en 2014 (YouTube, 2015).

Los anunciantes pueden aparecer en YouTube sirviéndose de varios sistemas para conseguir presencia y notoriedad de marca. Veamos la forma definida por la plataforma para que los anunciantes comuniquen sus mensajes. Después plantearemos por qué las marcas tienen interés en incluir a los nuevos influencers como parte de su estrategia de comunicación para determinadas campañas.

Existen tres grandes grupos de formatos en la plataforma: TrueView, Banners y Masthead.
Los TrueView se componen de tres tipos de anuncios que pertenecen a la sección de vídeo de Google Adwords. Los usuarios pueden interactuar con cada uno de ellos de manera distinta dependiendo del contexto en el que aparecen, por lo tanto, tienen cierto control sobre ellos. Para los anunciantes, estos anuncios tienen un coste por visualización (CPV), aunque a veces tan solo hace falta visualizar 30 segundos.

- Los anuncios in-stream normalmente se insertan antes del vídeo al que se intenta acceder, aunque en ocasiones pueden mostrarse en la mitad o al final. Existen dos tipos: los anuncios que se pueden saltar tras haber visualizado cinco segundos y los que el usuario debe ver por completo. Estos tipos de ads están activos para ordenadores, televisores, móviles y videoconsolas.

- Los anuncios in-video son pequeñas superposiciones de 480x70 píxeles en la parte inferior de los videos. Pueden estar en formato flash o solo texto y son semitransparentes para favorecer la integración. 
Únicamente están activos en ordenadores.

- Los anuncios in-display están representados por sugerencias de YouTube o vídeos promocionados y relacionados que aparecen a la derecha del video principal y sobre el resto de sugerencias no publicitarias. Sus medidas son de 300x250 o de 300x60 píxeles.

- Los anuncios in-search son muy parecidos a los anteriores, pero estos aparecen sobre los resultados de búsqueda de YouTube.

El siguiente grupo son los banners que aparecen en la plataforma, debido a que esta pertenece a la red de display de Google. En este caso, pueden mostrarse en cualquier página excepto en la principal, es decir, junto a los vídeos o en resultados de búsquedas, atendiendo a unas dimensiones de 300x250 o 300x60 píxeles. Los formatos válidos serán .GIF, .JPG, .PNG, .SWF, MP4, WebM u Ogg con un máximo de 24fps y $150 \mathrm{~Kb}$ o hasta $2,2 \mathrm{Mb}$ en los rich media. Las animaciones no pueden sobrepasar los 30 segundos ni tener sonido si el usuario no hace clic.
El último grupo es el compuesto por las diferentes clases de Masthead alojados en la parte superior de la página principal o homepage de Google. Es el formato más caro de la plataforma y por ello tiene una duración de 24 horas. Las dimensiones que lo forman son 970x250. Existen tres versiones:

- El Masthead Estándar es el más básico y tiene la opción de incluir un banner o un vídeo de YouTube en su interior de 300x250 píxeles.

- El Masthead Expandible se despliega a unas dimensiones de 970x500 píxeles.

- El Masthead de vídeo tiene unas dimensiones de 850x250 píxeles y cuenta con un vídeo que se auto reproduce solo hasta los 30 segundos y el usuario debe hacer clic para continuar.

Son muchas las marcas que utilizan la plataforma para crear canales o vídeos corporativos, de forma que obtienen presencia de marca gratuitamente. Pero ¿cómo es posible que en una plataforma en la que al día se visualizan millones de horas de contenido y existen más de mil millones de usuarios, una marca 
como Fox ronde tan solo el medio millón de suscriptores? Es una cifra que comparten actualmente grandes empresas internacionales como Universal Estudios o Coca Cola. Otras como Mercedes o Windows no alcanzan los 360.000 y varias como Desigual, Rolex o El Corte Inglés no superan los 40.000 .

YouTube es diferente, y si no se crean contenidos diseñados específicamente para su consumo en ella, la audiencia les da la espalda. Algunos lo han visto con claridad y son los que aportan contenido exclusivo para YouTube implicándose en la comunidad y manteniendo unos estándares de calidad, exclusividad, regularidad e interacción que se ven recompensados, por ejemplo, con 4.399.828 suscriptores en el caso de Red Bull o 2.501 .740 suscriptores en el de Apple.

Las redes sociales y la web 2.0 han ofrecido unas herramientas de colaboración que permiten a los usuarios interactuar de manera ágil, directa y continua. Internet se ha convertido en una comunidad de comunidades en las que, de manera ininterrumpida, entra y sale gente. Los miembros de dichas comunidades se sienten parte de los grupos a los que pertenecen y confían en los integrantes del colectivo. Y aquí aparece la gran oportunidad para las marcas: el $87 \%$ de los interrogados al respecto, considera clave las recomendaciones de gente cercana en sus compras. ¿Se puede considerar como gente cercana a los miembros de una comunidad digital? Pues posiblemente sí.

En la actualidad, y frente al descenso que sufre la publicidad convencional en términos de credibilidad, está demostrado que la alternativa más fiable para una buena estrategia de marketing es la generación de recomendaciones entre consumidores. (Castelló, 2010, p. 94)

Según un estudio de YouGov (2014), el 62\% de los usuarios dejan una opinión sobre los productos o servicios para ayudar a otros usuarios, y el 25\% opinan que es correcto ofrecer un feedback. Esta misma investigación determinó que el 90\% de los estadounidenses piensa que los comentarios son una parte importante en el proceso de compra, aunque esto no implique que siempre se dejen llevar por ellos.

Estos resultados muestran una fuerte corriente de atención de unos 
consumidores a otros y una pérdida de credibilidad por parte de las marcas.

En cualquier caso, es una realidad la dificultad de satisfacer y retener a los usuarios de la plataforma. Por ello, muchas marcas se han visto en la obligación de buscar alternativas dentro de la propia red. Y lo lógico es analizar el comportamiento de los personajes que obtenían una popularidad mucho mayor que ellas, que alcanzaban cifras de cinco, diez y hasta 37 millones de suscriptores. Individuos con targets, a veces muy definidos, que sabían qué querían ver e incluso más importante, de quién.

Se trata de los youtubers, el nuevo fenómeno de la red.

\section{El fenómeno youtuber Identidad}

El objetivo principal del equipo de YouTube Space es ayudar a los creadores a que su contenido sea increíble, gracias a programas estratégicos y talleres realizados en las instalaciones de producción de los YouTube Space de Los Ángeles, Nueva York, Londres, Tokio, São Paulo y Berlín.

A fecha de marzo de 2015, los creadores que habían grabado en un YouTube Space habían producido más de 10.000 vídeos que, a su vez, habían generado más de mil millones de reproducciones $\mathrm{y}$ más de 70 millones de horas de visualización. (YouTube, 2015)

Los propios implicados no se ponen de acuerdo al tratar de definir el fenómeno y sus protagonistas, por lo que es necesario recapitular para conseguir un concepto claro de la identidad de los youtubers.

El término aparece por primera vez en 2009, tras un informe que publicó Google con el título ¿Quiénes son los Youtubers? Dicho documento trataba de dibujar el perfil de sus usuarios, por tanto, únicamente hacía referencia a cualquier persona que utilizara la plataforma, tuviera una cuenta o no. Con el paso del tiempo, el concepto fue evolucionando para referirse únicamente a las personas que suben contenido original a YouTube. Pero en 2011, tras el lanzamiento del proyecto de partners, se incluyó la condición de recibir beneficios para designarte como tal.

Hoy en día, el término tiene una acepción parecida, pero con algunos matices. En 2013, el diario ABC lo definía como "ganarse la vida compartiendo videos". Un año más 
tarde, Antonio Rull, en un artículo publicado en eldiario.es, se refería al youtuber como "la persona que tiene cientos de miles de seguidores en su canal de YouTube y que pretende o puede ganarse la vida creando y subiendo esos videos a su canal".

Pablo León (2014), en El País, dijo que se trataba de "un joven que se dedica a grabar, colgar en Internet sus creaciones y vivir de ellas". Ana María Nimo (2015), en cambio, puntualizaba el término como "un self-made young man que ha sido capaz de cautivar con sus vídeos a una legión de seguidores".

De estas definiciones se obtienen unos rasgos que podrían bastar para identificar al "youtuber-tipo":

- Crea y sube videos con cierta regularidad a la plataforma.

- Es el protagonista del contenido.

- Tiene uno o varios canales abiertos.

- Acaudilla numerosos seguidores, de miles en adelante.

- Obtiene ingresos considerables, que le permiten vivir de ello o complementar sustancialmente sus ingresos principales.

- Es joven, pero no necesa riamente.
Es importante recalcar la idea de que el éxito que están teniendo varios personajes, de edades comprendidas entre los 20 y los 30 años, no debería limitar el alcance del término, puesto que un hombre de 40 años que trabaja en un bufete de abogados y pretende obtener un dinero extra subiendo vídeos a YouTube, no vive esencialmente de los ingresos de la plataforma, pero se le debe reconocer como youtuber.

\section{Evolución del personaje}

Parece ser que fue en Estados Unidos y México donde los jóvenes Ian Hecox y Anthony Padilla abrieron un canal y comenzaron a colgar videos grabados con webcams. Fue entre 2005 y 2007 cuando algunos nombres fueron adquiriendo popularidad, y a día de hoy prácticamente todos ellos siguen activos, algunos con más de 15 millones de suscriptores.

El contenido de los videos era diferente en cada uno de los canales. Algunos nos cuentan su día a día, bailan o cantan ante la cámara, parodian a personajes populares o políticos, o cuelgan grabaciones de algún evento o experiencia que consideren relevante o noticiable. 
Pero la circunstancia que pro- muchos jóvenes acudieron a la red dujo un crecimiento exponencial para ver juegos que no se habían pode los creadores en YouTube fueron dido comprar o pedir ayuda para los videojuegos. A raíz de la incor- completarlos. poración de este tipo de contenido,

\begin{tabular}{|c|c|c|c|c|c|c|}
\hline \multicolumn{3}{|c|}{ Filtrar por SB Puntuación } & \multicolumn{2}{|c|}{ Filtrar por más suscripciones } & Filtrar por Más vistos & \multirow{2}{*}{ VISTAS DE LOS VÍDEOS } \\
\hline RAX & SB & \multicolumn{2}{|c|}{ USUARIO } & \multicolumn{2}{|c|}{ - SUSCRIPTORES • } & \\
\hline 1 & & - & \#Music & 9187 & 1803 & - \\
\hline 2 & & & \#Gaming & 7789 & 9816 & - \\
\hline 3 & & 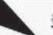 & \#Sports & 7630 & 3447 & \\
\hline 4 & 6269211 & D- & Pewdiepie & 4010 & 1934 & 10271398449 \\
\hline 5 & 6146160 & & \#Noticias & 3377 & 4723 & - \\
\hline 6 & 6121913 & & \#PopularOnYouTube & 2951 & 2624 & \\
\hline 7 & 188 & A & HolaSoyGerman. & 2477 & 6173 & 2218369411 \\
\hline 8 & 8791 & $B+$ & YouTube Spotlight & 23.0 & 20.791 & 618713857 \\
\hline 9 & 115 & A : & $\underline{\text { Smosh }}$ & 2136 & 8923 & 4908842628 \\
\hline 10 & 5343580 & - & Películas & 1856 & 0119 & \\
\hline 11 & 3508662 & c- & Pewdiepie & 4010 & 1962 & 10271398449 \\
\hline 12 & 36 & A+ & RihannaVEVo & 1774 & 8276 & 7388743096 \\
\hline 13 & 58 & A ! & OneDirectionVEVO & 1755 & 6625 & 4851031740 \\
\hline 14 & 47 & A+ & KatyPernyVEVo & 1712 & 3686 & 6339413125 \\
\hline 15 & 9 & $A^{+++}$ & TaylorSwiftVEVo & 16.7 & 21361 millones & 6779605985 \\
\hline 16 & 72 & A & EminemVEVo & 1662 & 8136 & 5553459131 \\
\hline 17 & 1063 & $B+$ & lennaMarbles & 1558 & 4793 & 1878065105 \\
\hline 18 & 596 & A- & nigahiga & 1546 & 7239 & 2400046175 \\
\hline 19 & 34 & A+ & VanossGaming & 1453 & 7289 & 3215382280 \\
\hline 20 & 33 & A+ & VanossGaming & 1453 & 7279 & 3215382280 \\
\hline 21 & 32 & A+ & VanossGaming & 1453 & 7276 & 3215382280 \\
\hline 22 & 71 & A. & laidefinichon & 1430 & 9740 & 2712271325 \\
\hline 23 & 25 & A+ & LustinBieberVEVo & 1391 & 1468 & 6399413828 \\
\hline 24 & 151 & A & Fine Brothers Entertainment & 1339 & 4465 & 3477720761 \\
\hline 25 & 22 & A+ : & TheEllenShow & 1289 & 8795 & 5150898884 \\
\hline 26 & 647 & A- & Machinima & 1267 & 3712 & 5536234270 \\
\hline 27 & 565 & A- & $\underline{E R B}$ & 1265 & 2300 & 1910017577 \\
\hline 28 & 6120862 & & \#PopMusic & 1236 & 2442 & \\
\hline 29 & 500 & A : & Yuya & 1194 & 4592 & 1141554364 \\
\hline 30 & 5966137 & & Programas de television & 1152 & 7306 & \\
\hline x4 & san & a & .el rinla MAimnomft & 1117 & $11 n 1$ & วัลกว10710 \\
\hline
\end{tabular}

Los treinta canales más vistos (Fuente: Social Blade) 

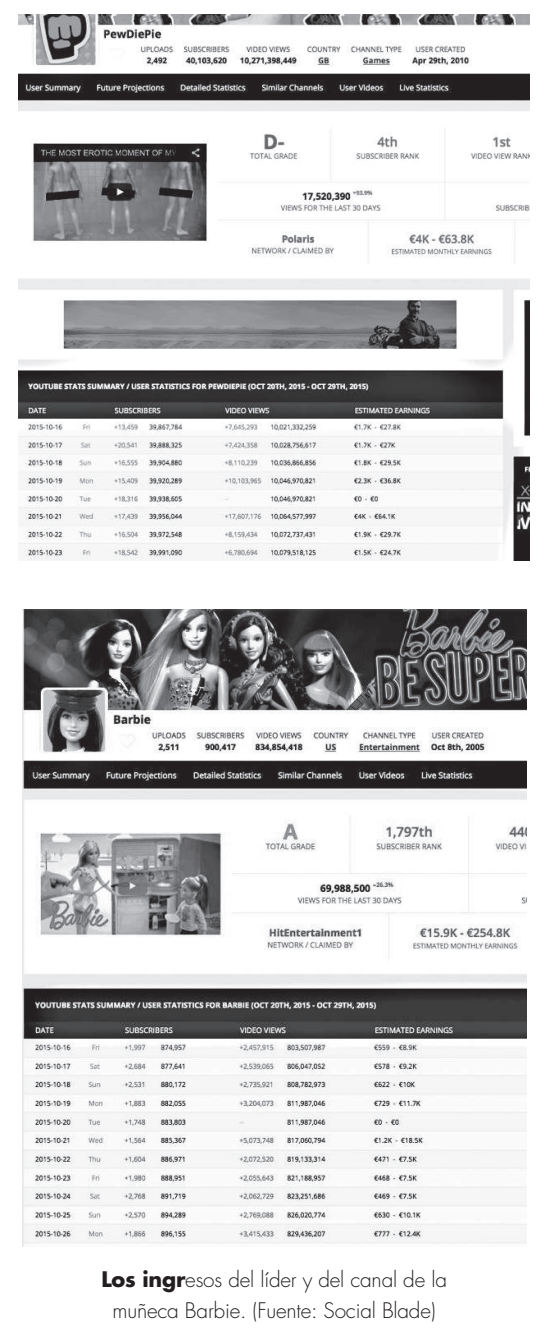

Al principio, muchos youtubers subían diferentes títulos sin incorporar su voz, únicamente con una pista de música o el audio del propio videojuego, pero al final todos terminaban integrándola, retroalimentándose de lo que veían en otros canales más atrevidos, normalmente procedentes de Estados Unidos.
Como se podía esperar, en Estados Unidos se profesionalizó la actividad mucho antes que en cualquier otro país. En España fue a partir de 2012 cuando el número de youtubers con un contrato ascendió un 150\%, pero en Estados Unidos, según aseguraba YouTube, ya había varios personajes que estaban ingresando más de un millón de dólares al año (Pareja, 2013).

A día de hoy, las cifras y repercusiones son desmesuradas, sobre todo para la industria del videojuego. Según el portal Social Blade, el sueco Felix Kjellber (PewDiePie), es el youtuber con mayor éxito del mundo. En su canal, donde sube de media dos vídeos diarios, realiza gameplays, vlogs, diferentes retos, videos de humor, etc. Como muchos youtubers, no tiene un perfil definido, aunque ha terminado especializándose en videojuegos. En Twitter tiene más de seis millones de seguidores y las cifras sobre sus ganancias oscilan entre 7 y 12 millones de dólares, según donde leamos la información: estas cifras nunca son fiables. La peculiaridad de este youtuber es el uso de un "chroma key" que le permite incluirse en la parte inferior izquierda de sus vídeos, aunque la integración no es profesional (cosa que nadie le va a exigir). 
El segundo canal con más visitas es el del chileno Germán Garmendia (HolaSoyGerman, 2015). En este caso, el contenido está únicamente enfocado a Vlogs de diferentes temáticas realizados de una manera humorística. Aunque existe variedad en sus temáticas, el estilo es muy lineal, acogiéndose a un plano medio corto en casi todos sus vídeos, gesticulando mucho y realizando muchos cortes. Al contrario que PewDiePie (2015), tiene un mayor número de visitas en los vídeos, con una media de 15 o 17 millones de visualizaciones, pero solo actualiza su portal dos veces al mes.

Actualmente, en España hay más de 28 youtubers que superan el millón de suscriptores, doce de ellos superan los dos millones y uno se encuentra el octavo en el top mundial en cuanto a número de suscriptores.

\section{La IPTV. El camino de la nueva televisión}

$\mathrm{Si}$ tomamos como referencia la evolución que ha tenido el consumo de audio en los últimos años y nos planteamos responder a estas preguntas: ¿Quién no usa Skype? ¿Quién no usa Spotify? ¿Cómo sigues tu emisora favorita?
La respuesta es: en Internet.

Todo el audio está en IP. Desde la BBC a la más pequeña emisora tienen su canal en streaming, en IP. Y si no puedes acceder en directo, están los podcasts.

Internet ha cambiado los hábitos de consumo y la generación de contenidos en el campo del audio.

¿Y el vídeo? A nivel de consumo, el IP está ya aquí y la frontera Ip/ Broadcast se está diluyendo rápidamente, lo cual supone:

- Mayor disponibilidad. Cada vez más herramientas e instalaciones trabajan sobre IP, los más diversos formatos, Internet, ADSL, 3G, 4G, Internet por satélite...

- Ahorro de costes. Son muy reducidos.

- Mayor flexibilidad. Soluciones versátiles y dinámicas, sin cableados rígidos.

- Mejor relación calidad-precio. ADSL en exteriores y switchers en estudios. De hecho, los equipos profesionales de televisión que se están fabricando ya están preparados para emitir por IP. 
La IP implica cableado (Rj45) y routers, y el envío de paquetes IP muchas veces no puede ser realizado por redes dedicadas a compartir tráfico. El retardo es una característica del IP y no es aceptable, pero solucionar estos problemas es cuestión de (poco) tiempo.

Por otra parte, la guerra entre los operadores de broadcast y los de telefonía móvil tiene todos los visos de resolverse a favor de estos últimos, con lo que la señal de televisión (TDT) quedaría relegada al satélite o a la IP.

La generalización de la fibra óptica en las ciudades se produce a marchas forzadas, y recibir velocidades de $300 \mathrm{MB}$ (por las que el $4 \mathrm{~K}$ viaja cómodamente), ya está al alcance de muchos espectadores. Este parece ser el camino y los distribuidores digitales de contenidos, como Netflix, lo tendrán más fácil, pero solo si las conexiones ADSL de alta velocidad comienzan a ser mayoritarias. Es cuestión de ancho de banda, y en España Movistar y otras compañías están comercializando los $300 \mathrm{MB}$ en su red de fibra, y gigantes como Apple o Amazon ya se preparan para emitir en streaming.

Es muy significativo que en Estados Unidos ya se maneja el término cord cutters en referencia a los que abandonan el sistema tradicional de ver la televisión (allí es el cable) para pasarse a la IPTV.

También hay que destacar el dato de que el consumo de vídeo por IP ha superado ya al herciano y no es el dato lo más importante, sino la tendencia, que sigue esa línea sin desviarse.

YouTube ya está técnicamente en posición de llevar a cualquier dispositivo calidades de UHDTV (de hecho, ya emite algunos vídeos en $4 \mathrm{~K})$. $Y$ eso significa que su supremacía no se va a limitar a las pequeñas pantallas de los jóvenes.

\section{La realidad supera al vocabulario}

Y, para terminar, una llamada de atención que abarca un campo mucho mayor que el inicialmente cubierto por este estudio.

La rapidez con que se están produciendo los cambios tecnológicos y sus consecuencias en el lenguaje audiovisual y las formas de su narrativa, están produciendo un laberinto de palabras de nuevo cuño, que inducen a una creciente confusión. Como afirma el profesor Carlos Scolari: 
¿Es correcto hablar de hipertelevisión? ¿Puede ser de utilidad el concepto de hipermediación? ¿Hasta cuándo el concepto digital nos servirá para diferenciar las nuevas formas de comunicación? Reconocer la existencia de un espacio de inestabilidad semántica y asumirla en nuestras conversaciones forma parte de nuestra responsabilidad en tanto investigadores de la Comunicación. (Scolari, 2015, p. 290)

Palabras o acrónimos como UHD, 4K, UHDTV, Full Ready, Alta Definición, etc., no tienen, según quien las use, el mismo significado. Un claro ejemplo de esto lo sufre cualquiera que se plantee estos días adquirir un televisor. Pocos serán los vendedores que le aclaren lo que significan algunos términos imprescindibles para decidirse por uno $\mathrm{u}$ otro modelo. $\mathrm{Y}$ a otro nivel, por ejemplo, durante el último IBC de Ámsterdam, un prestigioso ingeniero de la NHK japonesa nos hablaba de la resolución $8 \mathrm{~K}$ refiriéndose a ella como UHD...igual que al $4 \mathrm{~K}$.

Urge pues poner un poco de orden en este tema semántico, que se nos ha quedado demasiado analógico (término que ya está pasando a ser sinónimo de viejo).

\section{Conclusiones}

- Si algo queda claro después de analizar el fenómeno YouTube, es que no es algo temporal, sino que es el inicio de un nuevo modo de acceder a los contenidos audiovisuales. La juventud, principal consumidor de YouTube, está migrando de la televisión tradicional a las segundas y terceras pantallas, $y$ más concretamente a los canales de los youtubers.

- Las audiencias de estos canales empezaron a crecer en el 2012 y desde entonces no han cedido en su progresión. No se puede pronosticar en qué momento dejarán de aumentar, pero sí se puede confirmar que, a día de hoy, el fenómeno se encuentra en auge y cada día el alcance mediático de los creadores de contenido es mayor.

- Tanto es así, que las marcas y las grandes multinacionales están apostando por estos influencers. La relación que se establece entre ellos es mutua, debido a que las primeras les necesitan para llegar a nuevos targets y prescriptores por un precio menor que en cualquier otro medio convencional. Y los 
youtubers que no pueden vivir de las visitas, necesitan a las marcas para volcarse en el canal a tiempo completo.

- ¿Se puede vivir de YouTube? Sí, se puede. Pero para ello se debe tener una regularidad en el canal, contar con varios millones de visitas al mes y que el contenido tenga un buen CPM. Más de 30 youtubers obtienen más de 9 millones de visitas mensuales en España. En otros países es un trabajo estable.

- La consolidación de las redes IP en los núcleos urbanos y la previsible generalización del acceso a la Red mediante la fibra óptica por la que circulan los 300MB, en paralelo al arrinconamiento de la TDT por la telefonía $5 \mathrm{G}$ en el espectro radioeléctrico, hacen fácil adivinar cuál va a ser el lugar de cita que las generaciones futuras van a tener para informarse, entretenerse y educarse, no sé si por este u otro orden.

\section{Referencias}

Bassat, L. (1998). El libro rojo de la publicidad. Madrid: Espasa.
Burgess, J. y Green, J. (2013). YouTube: Online video and participatory culture. New York: John Wiley \& Sons.

Jenkins, H. (2006). Convergence culture: Where old and new media collide. New York: NYU Press.

Ordozgoiti, R. y de la Rica, R. O. (2010). Publicidad online: Las claves del éxito en Internet. ESIC Editorial. Scolari, C. (2008). Hipermediaciones. Elementos para una cultura digital interactiva. Barcelona: Gedisa.

Zunzarren, H., \& Gorospe, B. (2012). Guía del Social Media Marketing. ESIC Editorial.

\section{Artículos}

Castells, M. (2002). La dimensión cultural de Internet. FUOC.

Prensky, M. (2001). Nativos digitales, inmigrantes digitales. On the horizon, 9.

Roig, A. (2006). Prodúcete a ti mismo. Televisión, internet y la emergencia de la "cultura del clip".

Spira, J.B. (2003). Spam e-mail and its impact on it spending and productivity. Basex Report. 


\section{Páginas web}

Angermeier, M. Mapa conceptual de la Web 2.0 Recuperado de: http://museums.wikia.com/wiki/ File:Web20map.png

Anónimo. [LuzuVlogs]. (2015). YOUTUBE SPACE Los Angeles -

LuzuVlogs [Archivo de video] Recuperado de: https://www.youtube. com/watch? v=wKc30ZS8o-c

Antonio, R. Recuperado de: http://www.eldiario.es/autores/antonio_rull/ http://wwwlavanguardia.com/tecnologia/ internet/20141027/54417589877/ hitos-historia-bannelihtml

Collera, V. (2015). La revolución de losyoutubers. El País. Recuperado de: http://elpais.com/elpais/2015/06/ 24/eps/1435156615_876950.html

Comisión de formatos publicitarios. (2010). Más allá del Click Through Rate; Métricas de publicidad online. IAB Spain. Recuperado de: http:// www.iabspain.net/wp-content/ uploads/downloads/2012/02/IAB_ Más_allá_del_CTR_jul_2010.pdf

Comisión de redes sociales. (2015). Guía legal para Branded Content y figuras publicitarias afines. $I A B$
Spain. Recuperado de: http://wwW. iabspain.net/Wp-content/uploads/ downloads/2015/05/GUIA-BRANDED-CONTENT-IAB.pdf

De Cicco, J. (2008) YouTube: El archivo audiovisual de la memoria colectiva. Recuperado de: https:// www.academia.edu/2346783/YouTube_el_archivo_audiovisual_de_ la_memoria_colectiva

Díaz, E. (2015). Estadísticas mundiales 2015: Internet, social media y móvil. El Blog de Esmeralda DíazAroca. Recuperado de: http://www. esmeraldadiazarocacom/2015/05/ estadisticas-mundiales-2015 -internet.html

Digital Archive. (2014). En la publicidad display la eficacia es una cuestión de tamaño. Marketing Directo. Recuperado de: http://www.marketingdirecto.com/actualidad/digital/ en-la-publicidad-display-la-eficaciaes-una-cuestion-de-tamano/

Europa Press. (2013). Una nueva profesión: "Youtuber". ABC. Recuperado http://www.abc.es/tecnologia/redes/20130723/abci-profesion-youtuber- 201307231155.html García, L. (2003). ¿Web 2.0 vs Web 1.0? Boletín Electrónico de noticias 
de Educación a distancia. Recuperado en: http://www.raco. cat/ index.php/dim/article/viewFile/76637/98327.

HolaSoyGerman. Recuperado de: https://www.youtube.com/user/ HolaSoyGerman

IAB Spain. Recuperado de: http:// www.iabspain.net/wp-content/ uploads/downloads/20 l 5/0 l/Estudio_Anual_Redes_Sociales_ 2015 .pdf

León, P. (2014). De mayor quiero ser "youtuber". El País. Recuperado de: http://politica.elpais. com/politica/2014/11/14/actualidad/1415995907_985209.html

Media Response Group. (2007). Rising Star, los nuevos estilos de anuncios digitales que triunfan. Media Response Group. Recuperado de: http://www.mediaresponse.es/blog/ iab-rising-stars-los-nuevos-estilosde-anuncios-digitales-que-triunfan/ Naranjo, F. (2014). YouTube: Los 7 hitos que han marcado 7 años de su Vida. Puro Marketing. Recuperado de: http://www.puromarketing. com/15/13137/youtube-hitos-marcado-anos-vida.html
Nielsen Comunication. (2013). Estudio Nielsen sobre Advertising. Nielsen. Recuperado de: http:// www.nielsen.com/es/es/insi ghts/ news/2013/la-publicidad-con-humor-la-que-mas-cala.html

National Geographic. (2014). El nacimiento de Internet, lo más importante de los 90 según españoles. $\mathrm{Na}$ tional Geographic. Recuperado de: http://nationalgeographic.es/national-geographic-channel/lo-m-simportante-de-los-90

O'Reilly, T. (2005). What is Web 2.0 Recuperado de: http://www. oreillynet.com/pub/a/oreilly/tim/ news/2005/09/30/what-is-web20.html

Pareja, P. (2015). De profesión "Youtuber". El País. Recuperado de: http://cultura.elpais.com/ cultura/2013/10/06/television/13 81084045_173756.html

Sainz, K. (2014). Mientras en España se leen cada vez menos libros, los editores buscan lectores en YouTube. Vozpopuli. Recuperado de: http:// vozpopuli.com/ocio-y-cultura/ 55719-mientras-en-espana-se-leencada-vez-menos-libros-los-editoresbuscan-lectores-en-youtube 
Sanabre, C. (2014). 20 años de publicidad en internet (1994-2014). Educacionline. Recuperado de: http://www.educacionline.com/instituto-de-marketing-online/author/ csanabre/

Singel, R. (2013). OCT.27, 1994: Web gives birth to banner ads. Wired. Recuperado de: http://www.wired.com/2010/ 10/ 1027hotwiredbanner-ads/

SocialBlade. Recuperado de: http:// socialblade.com/youtube/top/category/education/mostsubscribed http://socialblade.com/youtube/ user/pewdiepie YouTube Estadísticas. Recuperado de: https://www.youtube.com/yt/ press/es/statistics.html 